\title{
SURPRISE, SURPRISE
}

Ronald Simon and colleagues report that oestrogen receptor $\alpha$ (ESR1) is commonly amplified in the early stages of proliferative breast disease, and that the detection of ESR1 copy number could provide clinically relevant information about treatment strategies for patients with breast cancer.

Using more than 2,000 breast cancer tissue samples to analyse ESR1 amplification by fluorescence in situ hybridization, they reported that ESR1 was amplified in $20.6 \%$ of these samples and that an additional $15.3 \%$ showed increases in ESR1 copy number. Low-grade mucinous cancers showed the highest rates of ESR1 amplifications (35.6\%), and benign papillomas and ductal hyperplasias also showed ESR1 amplification (36\% and $8.3 \%$, respectively), indicating that ESR1 amplification is a very early event in tumorigenesis and might have prognostic value.

Therefore, Simon and colleagues investigated the relationship between ESR1 amplification and the response to tamoxifen, which targets the oestrogen receptor. In a retrospective analysis of 175 breast cancer samples from patients who had undergone variable tamoxifen treatments, they showed that tamoxifen response correlated with and was dependent on ESR1 copy number. This is surprising for two reasons: first, this effect is the opposite to that of the androgen receptor in prostate cancer; and second, the importance of ESR1 amplification in breast cancer development and prognosis has not been detected until now.

ORIGINAL RESEARCH PAPER Holst F. et al. Estrogen receptor $\alpha$ (ESR1) gene amplification is frequent in breast cancer. Nature Genet. 8 April 2007 (doi: 10.1038/ ng2006)

\section{HIT AND MISS FOR CETUXIMAB}

The results from two large, randomized phase III trials of the monoclonal antibody cetuximab (Erbitux), which inhibits the epidermal growth factor receptor, have been announced.

One trial in more than 700 patients with pancreatic cancer, conducted by the Southwest Oncology Group, determined that giving cetuximab in combination with gemcitabine (compared with the administration of gemcitabine alone) as a first-line treatment option failed to improve overall survival. Pancreatic cancer currently has a 5-year survival rate of approximately $5 \%$, illustrating the limitations in treating this disease. As Martin Birkhofer, vice president of oncology global medical affairs at Bristol-Myers Squibb, states: “.... it is unfortunate that the use of Erbitux in this trial has not demonstrated the benefit it has shown in other tumours." Indeed, the second trial (called EXTREME), conducted in more than 400 patients with recurrent and/or metastatic squamous-cell carcinoma of the head and neck, showed that cetuximab in combination with 5-fluorouracil and platinum-based chemotherapy successfully improved the overall survival of these patients.

Encouraged by the EXTREME study, ImClone Systems intend to pursue the use of cetuximab in combinatorial regimens to treat patients with pancreatic cancer.

WEB SITES http://www.imclone.com; http://www.bms.com 